\title{
Letter to the Editor: Improving alpha spectrometry energy resolution by ion implantation with ICP-MS
}

\author{
Eduardo García-Toraño ${ }^{1}$
}

Received: 24 April 2015/Published online: 23 June 2015

(C) Akadémiai Kiadó, Budapest, Hungary 2015

Dear Dr. Revay,

I have read with very much interest the paper "Improving alpha spectrometry energy resolution..." (DOI 10.1007/ s10967-014-3500-8) that presents a new method to prepare alpha-particle spectrometry sources using ICP-MS instrumentation. While the method and experimental setup are remarkably well described, the paper fails, in my opinion, to provide sound evidences about the spectrometric quality of the sources. The basic problem is that the poor quality of the detectors used in the measurements does not allow a proper judgment of the source characteristics. As mentioned in several references given in the paper, detectors with an energy resolution below $9 \mathrm{keV}$ are available that could have been used to assess beyond any doubt the quality of sources. I am afraid that the use of detectors with significant lower resolutions $(21 \mathrm{keV})$ precludes the authors to conclude that their sources can match the performances of those prepared by other techniques such as vacuum evaporation. My comment should not be interpreted as a criticism to the process of preparation in which I am confident; it rather refers to the method used to assess the source quality.

Yours faithfully,

Dr. Eduardo García-Toraño

Radionuclides Metrology

CIEMAT

Avda. Complutense 40

28040 Madrid, Spain

This is a reply to the letter to the Editor of article doi 10.1007/s10967014-3500-8.

Eduardo García-Toraño

e.garciatorano@ciemat.es

1 CIEMAT, Madrid, Spain 